\title{
TRATAMENTO DA DEPRESSÃO COM ELETROCONVULSOTERAPIA (ECT) / ELETROCHOQUE: UMA REVISÃO SISTEMÁTICA DA LITERATURA
}

\author{
Luis Paulo Machado Scalise ${ }^{1}$ \\ Matheus Gonçalves Amarante Cruz ${ }^{2}$ \\ Marcelo Henrique Machado Scalise ${ }^{3}$
}

RESUMO: Introdução: Como é sabida, a depressão é considerada como grave problema de saúde pública, por produzir impactos bastante negativos na vida do paciente e também de toda a família e dessa forma compromete os diversos aspectos pessoal, social e também laboral. Existem diversos tipos de tratamento, dentre eles o medicamentoso, a Terapia Cognitivo Comportamental (TCC) e a Eletroconvulsoterapia (ECT) ou também conhecida como Eletrochoque. A ECT abarca a indução de uma convulsão para fins terapêuticos a partir da administração de um choque de estímulo elétrico de frequência variável. Esta última e também objeto de estudo foi por longa data estigmatizada. Objetivo: Verificar por meio de uma revisão sistemática a importância e eficiência da Eletroconvulsoterapia (ECT) no tratamento da Depressão. Métodos. Trata-se de uma revisão sistemática da literatura, na qual foram analisadas as seguintes etapas: identificação da questão da pesquisa, busca na literatura, categorização dos estudos, avaliação dos estudos, interpretação dos resultados e síntese do conhecimento. Optou-se pela base de dados pela base de dados National Library of Medicine e National Institutes of Health (MEDLINE), acessada por meio do sistema PubMed. A estratégia de busca utilizou as seguintes combinações de palavras-chave: A estratégia de busca utilizou as seguintes combinações de palavras-chave: "Depression"; "Electroconvulsive therapy"; "Electroshock"; "Strategies"; "Psychiatry"”. Resultados. Fizeram parte do escopo desta revisão Io artigos que demonstraram ter relação intrínseca em seus resultados quanto à eficácia da ECT no tratamento da depressão. Conclusão. Apesar das limitações encontradas (número ínfimo de artigos encontrados na língua corrente que abarcavam os descritores) concluiu-se que a depressão é problema de saúde pública e acomete, inclusive, o transtorno depressivo maior (TDM) já é considerado uma das principais doenças incapacitantes do mundo, afetando aproximadamente 350 milhões de pessoa. Concluiu-se ainda ser a ECT opção de tratamento muito eficaz e deve ser disponível para pacientes selecionados.

Palavras-chave: Depressão. Eletroconvulsoterapia. Eletrochoque. Estratégias. Psiquiatra.

\footnotetext{
'Graduação em Medicina pela Universidade Brasil. Email: dr.luispauloscalise@hotmail.com

${ }^{2}$ Graduação em Medicina pela Universidade Brasil

${ }^{3}$ Graduado em medicina pela Universidade Camilo Castelo Branco.
} 
ABSTRACT: Introduction: As it is known, depression is considered a serious public health problem, as it produces very negative impacts on the patient's life and also on the whole family, thus compromising the various personal, social and labor aspects. There are several types of treatment, including medication, Cognitive Behavioral Therapy (CBT) and Electroconvulsive Therapy (ECT) or also known as Electroshock. ECT encompasses the induction of a seizure for therapeutic purposes from the administration of a variable frequency electrical stimulus shock. The latter, and also the object of study, has been stigmatized for a long time. Objective: To verify through a systematic review the importance and efficiency of Electroconvulsive Therapy (ECT) in the treatment of Depression. Methods: This is a systematic literature review, in which the following steps were analyzed: identification of the research question, literature search, categorization of studies, evaluation of studies, interpretation of results and synthesis of knowledge. The National Library of Medicine and National Institutes of Health (MEDLINE) database was chosen, accessed through the PubMed system. The search strategy used the following keyword combinations: The search strategy used the following keyword combinations: "Depression"; "Electroconvulsive therapy"; "Electroshock"; "Strategies"; "Psychiatry"”. Results: The scope of this review included Io articles that showed an intrinsic relationship in their results regarding the effectiveness of ECT in the treatment of depression. Conclusion: Despite the limitations found (minimal number of articles found in the current language that encompassed the descriptors), it was concluded that depression is a public health problem and affects, even, major depressive disorder (MDD) is already considered one of the main disabling diseases of the world, affecting approximately 350 million people. It was further concluded to be a very effective treatment option and should be available to selected patients.

Keywords: Depression. Electroconvulsive therapy. Electroshock. Strategies. Psychiatrist.

\section{INTRODUÇÃO}

Atualmente, há uma estimativa que 300 milhões de pessoas sofram de depressão em termos mundiais. Este número é o equivalente a 4,4\% da população. A brasileira é tida como a mais depressiva da América Latina, com um percentual de 5,8\% acometido. Mulheres mais afetadas, e quando estão em situação de vulnerabilidade, pobreza, desemprego, doença e uso de álcool e drogas ficam o risco é mais potencializado (ASSIS et al., 2020).

A fim de um entendimento mais afincado de termos que serão tratados neste artigo, tal como "depressão" e "eletroconvulsoterapia”, de antemão serão feitas algumas considerações sobre referida patologia e também da estratégia de tratamento utilizada. Pode-se dizer que a depressão é uma das patologias mais faladas e discutidas nos dias atuais e pode ocasionar a destruição de uma vida. Muitas pessoas acham que é “meramente" frescura, mas os estudos comprovam que é, de fato, uma patologia e se não 
tratada pode levar à morte uma pessoa. Em termos estatísticos é, aproximadamente, uma em cada cinco pessoas que são acometidas pela depressão (MATOS; OLIVEIRA, 2013).

A palavra "depressão" indica uma série de alterações de ordem comportamental, emocional que atingem de forma direta a vida social e privada de uma pessoa ocasionando desconforto, restrições e até mesmo prejuízos da vida da pessoa que está acometida pela depressão, pois faz com que ela tenha uma visão "distorcida” da realidade. Depressão não é a mesma coisa de tristeza ou sofrimento, pois não algo rotineiro de uma pessoa saudável e também provida de confiança (MATOS; OLIVEIRA, 2013).

Com relação à causa que origina o TDM os estudos apontam como ainda não completamente conhecida, no entanto, sugerem que a doença possui fisiopatologia multifatorial. Estados depressivos graves frequentemente ocorrem sem que haja a presença de um fator psicobiológico desencadeante evidente. Há evidência de que fatores somáticos, genéticos e ambientais estejam envolvidos com a doença, destacando-se principalmente distúrbios na função hipotalâmica e de neurotransmissão. Uma hipótese é que ocorra disfunção na transmissão monoaminérgica, reduzindo a neurotransmissão de serotonina (5-HT), norepinefrina (NA) e dopamina (DA) (ARAÚJO et al., 2009).

Sobre a sua manifestação Araújo et al., (2009) afirma que pode ocorrer como episódio depressivo maior (EDM). E os critérios definidos pelo DSM-V são caracterizados por cinco sintomas: humor, interesse ou prazer reduzido, perda ou ganho de peso, insônia ou hipersonia, agitação ou retardo motor, fadiga ou perda de energia, sensação de inutilidade, culpa excessiva ou inapropriada, capacidade diminuída para pensar ou concentrar-se, indecisão e pensamentos recorrentes sobre morte. São três os critério que abarcam dois dos três sintomas fundamentais e que são: humor deprimido, perda de interesse ou prazer e energia reduzido. Outros sintomas podem acompanhar, dentre eles podem ser citados: diminuição da concentração e atenção, da autoestima, da autoconfiança associados a perdas de ordem funcional ou até mesmo social.

O diagnóstico é necessário e perpassa diversas fases que vai desde uma anamnese bem delineada e planejada com o paciente e se necessário até mesmo com os seus familiares e/ou cuidadores, exame psiquiátrico meticuloso, exame clínico geral, avaliação neurológica, identificação de efeitos adversos de medicamentos, exames laboratoriais e de 
neuroimagem. Seguindo esses passos o diagnóstico da depressão será o mais preciso possível (MATOS; OLIVEIRA, 2013).

Os sintomas são ligados, normalmente, às doenças físicas ou também à utilização de medicamentos. Faz-se oportuno mostrar tais sintomas através do quadro a seguir:

Quadro r: Sintomas da depressão

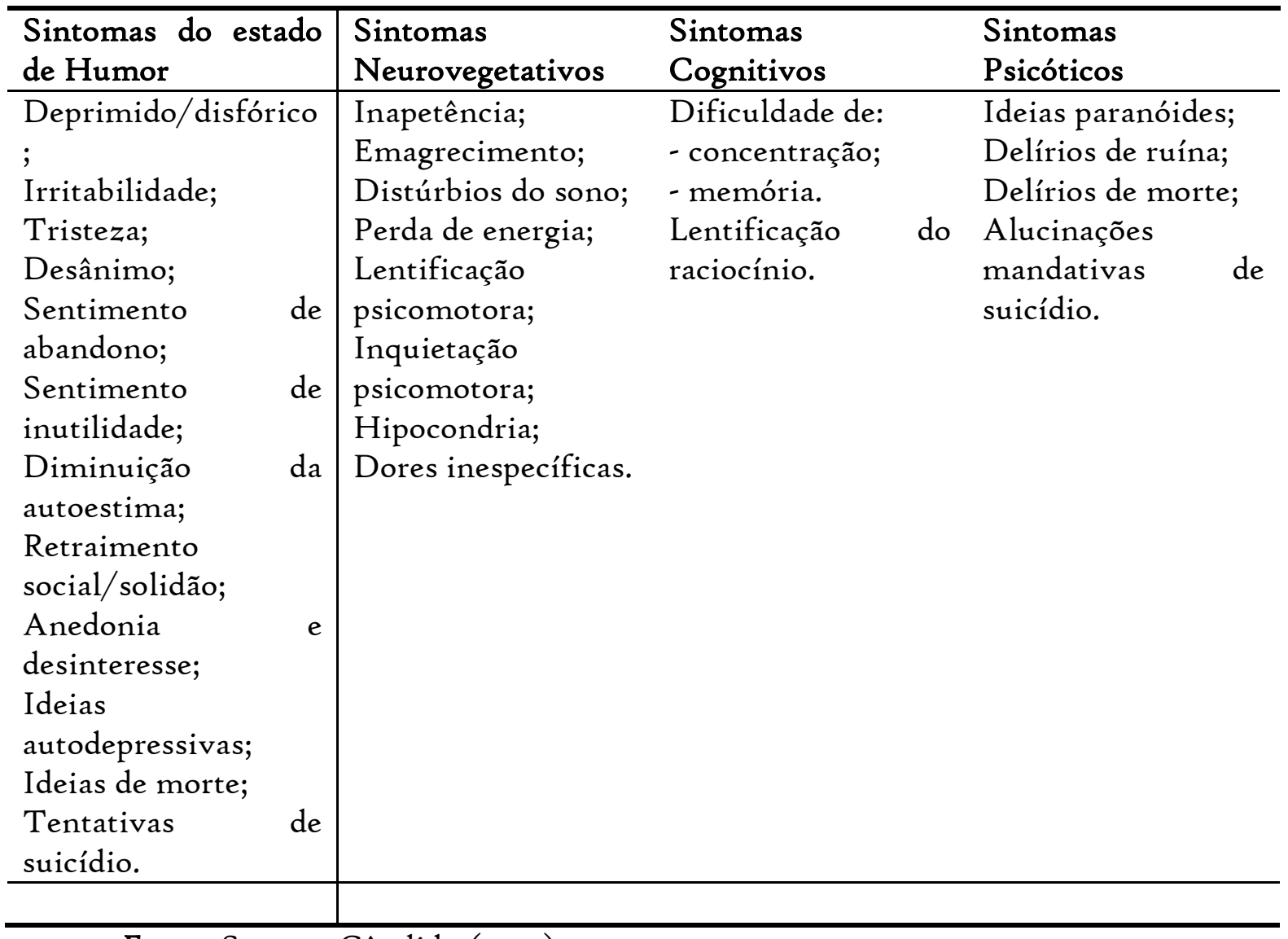

Fonte: Souza e Cândido (2009)

A depressão não tem uma idade exata ou fixa para se manifestar, ou seja, ela pode acontecer em qualquer fase da vida de uma pessoa com qualquer idade, e ela tem sua classificação delimitada de acordo com as fases em que a depressão aparece. De acordo com Bauer et al., (2009) referidos transtornos podem ser classificados como: transtorno depressivo-maior (TDM), também conhecido como depressão unipolar e transtorno unipolar. Este transtorno acomete mais mulheres que homens. Inicia, geralmente, aos 40 anos idade o que não impede que ela acometa mulheres em qualquer outra idade. Os fatores genéticos influenciam no surgimento do transtorno depressivo. Com relação ao tratamento da referida patologia vai depender da gravidade em que ela se apresenta. Mas é 
sabido que há muitas formas e variadas terapias que objetivam conter a depressão (BAUER, 2009).

A partir de uma linha de visão cognitiva sobre o referido transtorno, pode-se considerar que a depressão é resultante de é resultante de hábitos de pensamento extremante arraigado. Sendo assim, os comportamentos negativos são decorrência de pensamentos e crenças disfuncionais, ao invés de forças inconscientes, como sugere a teoria freudiana. Assim sendo, ela pode ser compreendida como consequência das próprias cognições e de diagramas desadaptativos, não descartando o pressuposto de que as causas da depressão são multifatoriais, envolvendo fatores biológicos, socioculturais e psicológicos (PALOSKI; CHRISTI, 2014).

A literatura sugere que o seu tratamento não seja apenas direcionado com o uso de antidepressivos, considerando que ele deverá ter como objetivo primordial a melhora da qualidade de vida do paciente, diminuindo a necessidade de internação hospitalar, contenção do suicídio, diminuindo também reincidências de quadros depressivos graves e assim garantindo uma adesão boa com efeitos adversos mínimos (ROCHA ET AL., 2016).

Os mesmos autores considerando todo o sustentáculo terapêutico disponível, outras alternativas, como a eletroconvulsoterapia, principalmente, quando a depressão é resistente ao tratamento por pelo ao menos, dois ensaios adequados de farmacoterapia antidepressiva. A ECT também conhecida como eletrochoque, é definida como um tratamento psiquiátrico, no qual são provocadas atividades elétricas do cérebro, induzidas por corrente elétrica. O número de sessões e de aplicações é individual e definido pelo médico psiquiátrico. É tido como ato privativo do médico e regulamentado pelo Conselho Federal de Medicina (MACHADO et al., 2019).

A eletroconvulsoterapia (ECT) é amplamente considerada o tratamento mais eficaz para a depressão e é usada com mais frequência quando os medicamentos antidepressivos não produziram melhora clínica adequada, ou seja, no tratamento depressão resistente (TRD) definida como falha em responder a dois ou mais testes adequados de medicamentos antidepressivos (ZHANDE et al., 2015).

Importa ainda ressaltar que a ECT é um dos procedimentos mais usados em medicina, embora ainda seja alvo de controvérsias. De maneira técnica, ela consiste na passagem de um choque elétrico pelo cérebro de uma pessoa por meio do uso de eletrodos 
inseridos na superfície do crânio. A corrente elétrica gera diversos impulsos motores que se manifestam por meio de convulsões e estas são vistas como antagonistas de sintomas psicóticos o que se fundamenta na antiga observação da epilepsia, uma doença caracterizada de maneira mais convencional por convulsões (OLIVEIRA, 2019).

$\mathrm{Na}$ época de sua criação, década de 1930 gerou certa celeuma em decorrência dos possíveis efeitos danosos que ela poderia ocasionar e por tais motivos entre 1960 e 1970 a ECT passou a ser alvo de polêmica e denúncias em todo campo da saúde mental e medicina. Hoje é visto com perspectivas diversas com direcionamento de uma proposta terapêutica diversa à medicamentosa, mas ainda existem oposições (OLIVEIRA, 2019).

Inclusive ressalta Alves (2016 apud Muniz et al., 2020) que diversos estudos tem sido desenvolvidos a fim de comprovar a eficácia da ECT no tratamento de sintomas depressivos, sendo essa eficácia medida a partir dos tempos de duração da convulsão. Tanto as invasivas quanto não invasivas tem mostrado efeito significativo na neurogênese e na modulação da plasticidade cerebral, modificando o paradigma do foco bioquímico (medicamentoso) para o biofísico (estímulo cerebral direito).

Feitas tais considerações justificando a importância na escolha do referido assunto, importa dizer que o objetivo geral do presente trabalho consiste em avaliar a importância e eficiência da Eletroconvulsoterapia (ECT) no tratamento da Depressão. Face às assertivas anteriormente expostas, esta investigação tem como pergunta norteadora: "A ECT é eficaz no tratamento dos sintomas da depressão?”

Em relação estreita ainda às assertivas anteriormente elencadas importa dizer que é de grande valia a pesquisa em questão a fim de investigar sobre a eficácia de um método permeado de estigmas, ainda que validado pelo Conselho Federal de Medicina. Diante disso, o objetivo desta revisão sistemática é levantar as publicações que trazem a utilização da ECT e resultados exitosos.

\section{MATERIAIS E MÉTODOS \\ 2.1 Classificação da Pesquisa}

Trata-se de uma revisão sistemática da literatura, na qual foram analisadas as seguintes etapas: identificação da questão da pesquisa, busca na literatura, categorização dos estudos, avaliação dos estudos, interpretação dos resultados e síntese do conhecimento. Revista Ibero-Americana de Humanidades, Ciências e Educação. São Paulo, v.7.n.10. out. 2021. 
A questão norteadora da revisão de literatura consistiu em: “A eletroconvulsoterapia (ECT) ou eletrochoque é eficiente ou não no tratamento da depressão?”.

\subsection{Base de Dados}

Foi usada a base de dados National Library of Medicine e National Institutes of Health (MEDLINE), acessada por meio do sistema PubMed. Para os resultados foram analisados os mais relevantes estudos publicados originalmente na língua inglesa, dos dez últimos anos, ou seja, a partir de 2orr. Com objetivo de selecionar os estudos de maior evidência científica, contemplamos somente estudos que tiveram práticas evidenciadas, identificados por revisões anteriores, e que tiveram seu escopo atualizado e revisado.

\subsection{Extração de Dados e Identificação de Permanência}

A fim de identificar os estudos que contemplariam os resultados, foram lidos em primeiro momento títulos, resumos e palavras-chave:
a) Depressão;
b) Eletroconvulsoterapia;
c) Eletrochoque;
d) Estratégias;
e) Psiquiatria.

\subsection{Estratégia de Busca}

A estratégia de busca utilizou as seguintes combinações de palavras-chave: "Depression”; "Electroconvulsive therapy”; “Electroshock”; "Strategies"; "Psychiatry””.

\subsection{Critérios de Elegibilidade \\ 2.5.I Critérios de Inclusão}

Os critérios de inclusão postos para esta revisão foram: estudos envolvendo seres humanos maiores de 18 anos, publicados no período de 2011 a 2011 em inglês e português (que obtivemos um número ínfimo). 


\subsubsection{Critérios de exclusão}

Além disso, estudos sem resumo disponível; artigos do tipo: revisões bibliográficas não sistematizadas, cartas, resenhas, editoriais, reflexões, publicações do tipo: livros; capítulos de livros; publicações governamentais; boletins informativos; teses; dissertações; monografias e trabalhos de conclusão de curso.

\section{RESULTADOS}

Foram identificados 27 estudos envolvendo a importância da Eletroconvulsoterapia no tratamento da depressão. Após aplicação dos critérios de inclusão e exclusão restaram Io. $\mathrm{Na}$ tabela I, 2 e 3 apresentamos uma síntese dos trabalhos selecionados e revisado Tabela I. Síntese dos estudos e seus principais resultados

\begin{tabular}{|c|c|c|c|c|}
\hline Autor/Ano. & $\begin{array}{l}\text { Rapinesi et } \\
\text { al., (2013) }\end{array}$ & $\begin{array}{l}\text { Hermida et al., } \\
\qquad(2018)\end{array}$ & $\begin{array}{l}\text { Ma et al., } \\
(2020)\end{array}$ & $\begin{array}{l}\text { Santos Junior et } \\
\text { al., (2013) }\end{array}$ \\
\hline Objetivo & $\begin{array}{l}\text { Analisar o } \\
\text { caso de um } \\
\text { jovem } \\
\text { paciente com } \\
\text { depressão } \\
\text { refratário a } \\
\text { medicament } \\
\text { o e que } \\
\text { respondeu } \\
\text { prontamente } \\
\text { à ECT }\end{array}$ & $\begin{array}{c}\text { Fornecer aos } \\
\text { psiquiatras uma } \\
\text { visão equilibrada e } \\
\text { aprofundada dos } \\
\text { avanços recentes } \\
\text { na técnica de } \\
\text { ECT, bem como } \\
\text { as evidências da } \\
\text { ECT para o } \\
\text { tratamento da } \\
\text { depressão, } \\
\text { incluindo TDM } \\
\text { em pacientes com } \\
\text { problemas } \\
\text { médicos } \\
\text { comórbidos. }\end{array}$ & $\begin{array}{l}\text { Examinar a } \\
\text { eficácia da } \\
\text { ECT em uma } \\
\text { amostra de } \\
\text { tratamento do } \\
\text { mundo real } \\
\text { em um } \\
\text { hospital } \\
\text { psiquiátrico } \\
\text { chinês que } \\
\text { incluiu } \\
\text { pacientes } \\
\text { resistentes e } \\
\text { não resistentes } \\
\text { ao tratamento. }\end{array}$ & $\begin{array}{l}\text { Descrever o perfil } \\
\text { sociodemográfico } \\
\text { e clínico de } \\
\text { pacientes } \\
\text { submetidos à } \\
\text { eletroconvulsotera } \\
\text { pia (ECT) em um } \\
\text { hospital geral } \\
\text { universitário }\end{array}$ \\
\hline $\begin{array}{l}\text { Tipo do } \\
\text { estudo }\end{array}$ & $\begin{array}{l}\text { Relato de } \\
\text { Caso }\end{array}$ & Bibliográfico & $\begin{array}{c}\text { Estudo } \\
\text { observacional }\end{array}$ & $\begin{array}{l}\text { Estudo } \\
\text { retrospectivo }\end{array}$ \\
\hline
\end{tabular}




\begin{tabular}{|c|c|c|c|c|}
\hline Protocolo & $\begin{array}{c}\text { Dose de } \\
\text { carga de } \\
\text { 176,4 e ECT } \\
\text { em dias } \\
\text { alternados } \\
\text { por } 6 \\
\text { sessões. }\end{array}$ & $\begin{array}{l}\text { Sugerem que o uso } \\
\text { da ECT deve ser } \\
\text { individualizado de } \\
\text { acordo com cada } \\
\text { população especial }\end{array}$ & $\begin{array}{c}\text { Os } \\
\text { tratamentos } \\
\text { de ECT no } \\
\text { Guangzhou } \\
\text { Huiai } \\
\text { Hospital são } \\
\text { programados } \\
\text { três vezes por } \\
\text { semana } \\
\text { usando o } \\
\text { dispositivo } \\
\text { Mecta } \\
\text { Spectrum sooo } \\
\text { (Mecta Corp). } \\
\text { Após a pré- } \\
\text { oxigenação } \\
\text { com oxigênio } \\
\text { a roo\%, a } \\
\text { anestesia com } \\
\text { propofol (ou } \\
\text { etomidato se } \\
\text { clinicamente } \\
\text { indicado) é } \\
\text { combinada } \\
\text { com o } \\
\text { relaxante } \\
\text { muscular. }\end{array}$ & $\begin{array}{c}\text { Os protocolos } \\
\text { foram levantados } \\
\text { por meio de } \\
\text { entrevistas. Não } \\
\text { foi questionado o } \\
\text { tipo de protocolo e } \\
\text { se o paciente } \\
\text { havia sido } \\
\text { submetido à ECT. }\end{array}$ \\
\hline $\begin{array}{l}\text { Principais } \\
\text { resultados }\end{array}$ & $\begin{array}{c}\text { Melhora } \\
\text { significativa } \\
\text { nos sintomas } \\
\text { depressivos } \\
\text { maiores }\end{array}$ & $\begin{array}{c}\text { Embora a ECT } \\
\text { tenha sido } \\
\text { estigmatizada } \\
\text { historicamente, a } \\
\text { prática atual da } \\
\text { ECT continua a } \\
\text { melhorar por meio } \\
\text { de empolgantes } \\
\text { pesquisas de } \\
\text { ponta. }\end{array}$ & $\begin{array}{l}\text { Em pacientes } \\
\text { com depressão } \\
\text { mais grave, a } \\
\text { ECT se } \\
\text { mostrou mais } \\
\text { eficiente. }\end{array}$ & $\begin{array}{l}\text { As principais } \\
\text { indicações para } \\
\text { ECT foram } \\
\text { depressão e } \\
\text { catatonia. } \\
\text { Complicações } \\
\text { foram observadas } \\
\text { em menos de } \\
\text { metade dos } \\
\text { casos, e a maioria } \\
\text { delas teve caráter } \\
\text { temporário e não } \\
\text { grave. }\end{array}$ \\
\hline
\end{tabular}




\begin{tabular}{|c|c|c|c|c|}
\hline Conclusão & $\begin{array}{c}\text { A ECT pode } \\
\text { ser uma } \\
\text { alternativa } \\
\text { eficaz no } \\
\text { tratamento } \\
\text { da depressão. }\end{array}$ & $\begin{array}{l}\text { - A ECT continua } \\
\text { sendo uma } \\
\text { modalidade de } \\
\text { tratamento } \\
\text { importante } \\
\text { altamente eficaz } \\
\text { no tratamento da } \\
\text { depressão maior. }\end{array}$ & $\begin{array}{l}\text { Apesar da } \\
\text { eficiência da } \\
\text { ECT para } \\
\text { depressão } \\
\text { grave, o } \\
\text { estudo foi } \\
\text { concluído } \\
\text { sugerindo } \\
\text { mais estudos } \\
\text { seriam } \\
\text { necessários } \\
\text { para } \\
\text { identificar o } \\
\text { nível } \\
\text { específico de } \\
\text { gravidade dos } \\
\text { sintomas em } \\
\text { que a ECT se } \\
\text { torna benéfica. }\end{array}$ & $\begin{array}{c}\text { A ECT foi } \\
\text { realizada de } \\
\text { acordo com } \\
\text { diretrizes } \\
\text { internacionais. Na } \\
\text { grande maioria } \\
\text { dos casos, efeitos } \\
\text { indesejáveis } \\
\text { foram } \\
\text { temporários e não } \\
\text { graves. A resposta } \\
\text { à ECT foi positiva } \\
\text { na maioria dos } \\
\text { casos, } \\
\text { especialmente em } \\
\text { pacientes } \\
\text { catatônicos. }\end{array}$ \\
\hline
\end{tabular}

Tabela 2. Síntese dos estudos e seus principais resultados

\begin{tabular}{|c|c|c|c|c|}
\hline Autor/Ano. & $\begin{array}{l}\text { Antunes et } \\
\text { al., (2019) }\end{array}$ & $\begin{array}{c}\text { Zhand et al., } \\
\text { (2015) }\end{array}$ & Li et al., (2020) & $\begin{array}{c}\text { Rönnqvist et al., } \\
(202 \mathrm{I})\end{array}$ \\
\hline Objetivo & $\begin{array}{c}\text { O objetivo do } \\
\text { estudo foi } \\
\text { demonstrar o } \\
\text { papel da ECT } \\
\text { no } \\
\text { tratamento da } \\
\text { depressão e } \\
\text { destacar } \\
\text { aspectos } \\
\text { moderno } \\
\text { relativo à sua } \\
\text { prática. }\end{array}$ & $\begin{array}{c}\text { Objetivou } \\
\text { apresentar uma } \\
\text { série de casos } \\
\text { abrangente de } \\
\text { adolescentes que } \\
\text { receberam a ECT } \\
\text { para tratamento } \\
\text { da depressão } \\
\text { resistente. }\end{array}$ & $\begin{array}{c}\text { Interpretar } \\
\text { estudos } \\
\text { clínicos e pré- } \\
\text { clínicos de } \\
\text { ECT e seu } \\
\text { potencial sobre } \\
\text { a depressão. }\end{array}$ & $\begin{array}{l}\text { Determinar a } \\
\text { associação entre } \\
\text { ECT e o risco de } \\
\text { suicídio em } \\
\text { pacientes com } \\
\text { transtorno } \\
\text { depressivo maior } \\
\text { unipolar. }\end{array}$ \\
\hline $\begin{array}{l}\text { Tipo do } \\
\text { estudo }\end{array}$ & $\begin{array}{l}\text { Revisão da } \\
\text { literatura }\end{array}$ & $\begin{array}{l}\text { Revisão de } \\
\text { prontuários }\end{array}$ & $\begin{array}{l}\text { Revisão da } \\
\text { literatura }\end{array}$ & Estudo de \\
\hline Protocolo & $\begin{array}{c}\text { Questionário } \\
\text { s a pacientes } \\
\text { submetidos a } \\
\text { ECT }\end{array}$ & $\begin{array}{c}\text { Adolescentes que } \\
\text { fizeram ECT por } \\
5 \text { anos um } \\
\text { hospital } \\
\text { psiquiátrico }\end{array}$ & & $\begin{array}{c}\text { Este estudo de } \\
\text { coorte usou dados } \\
\text { obtidos de } \\
\text { registros } \\
\text { nacionais suecos e }\end{array}$ \\
\hline
\end{tabular}




\begin{tabular}{|c|c|c|c|c|}
\hline & & $\begin{array}{c}\text { Canadense. } \\
\text { Receberam uma } \\
\text { média de I4 (SD, } \\
4,5 \text { ) sessões de } \\
\text { ECT por } \\
\text { paciente }\end{array}$ & & $\begin{array}{c}\text { foi relatado de } \\
\text { acordo com o } \\
\text { Relatório de } \\
\text { Fortalecimento do } \\
\text { Relatório de } \\
\text { Estudos } \\
\text { Observacionais } \\
\text { em Epidemiologia } \\
\text { (STROBE) } \\
\text { diretriz. }\end{array}$ \\
\hline $\begin{array}{l}\text { Principais } \\
\text { resultados }\end{array}$ & $\begin{array}{l}\text { Os principais } \\
\text { achados desta } \\
\text { revisão } \\
\text { foram: I) } \\
\text { ECT é mais } \\
\text { efetiva do que } \\
\text { qualquer } \\
\text { medicação } \\
\text { antidepressiv } \\
\text { a; 2) a } \\
\text { remissão da } \\
\text { depressão } \\
\text { com a ECT } \\
\text { varia, em } \\
\text { geral, de 5o a } \\
\text { 8o\%; } 3 \text { ) Ainda } \\
\text { é controverso } \\
\text { o efeito } \\
\text { da } \\
\text { eletroconvuls } \\
\text { oterapia nos } \\
\text { níveis de } \\
\text { fator } \\
\text { neurotrófico } \\
\text { derivado do } \\
\text { cérebro (acho } \\
\text { que aqui pode } \\
\text { colocar entre } \\
\text { parenteses ao } \\
\text { "BNDF"); } \\
4 \text { ) } \\
\text { eletroconvuls } \\
\text { oterapia com } \\
\text { efeito } \\
\text { positivo na } \\
\text { melhora da }\end{array}$ & $\begin{array}{l}\text { Os resultados } \\
\text { apontaram } \\
\text { melhora } \\
\text { significativa em } \\
77 \% \text { dos } \\
\text { adolescentes que } \\
\text { receberam a } \\
\text { ECT. }\end{array}$ & $\begin{array}{l}\text { Os resultados } \\
\text { apontam ser a } \\
\text { ECT } \\
\text { inofensiva e } \\
\text { eficaz, a taxa } \\
\text { de aplicação } \\
\text { ainda está } \\
\text { baixo e é } \\
\text { considerado o } \\
\text { último recurso } \\
\text { no tratamento } \\
\text { depressão. }\end{array}$ & $\begin{array}{l}\text { A } \\
\text { eletroconvulsotera } \\
\text { pia foi } \\
\text { significativament } \\
\text { e associado a uma } \\
\text { diminuição do } \\
\text { risco de suicídio } \\
\text { em pacientes com } \\
\text { características } \\
\text { psicóticas (HR, } \\
\text { o,20; } \\
\text { IC de 95\%, o,o8- } \\
\text { o,54) e aqueles } \\
\text { com idade entre } 45 \\
\text { a } 64 \text { anos (HR, } \\
\text { o,54; IC de 95\%, } \\
\text { o,30-o,99) ou 65 } \\
\text { anos ou } \\
\text { mais velhos (HR, } \\
\text { o,30; IC de 95\%, } \\
\text { o,15-o,59), mas não } \\
\text { em pacientes com } \\
44 \text { anos ou mais } \\
\text { jovens (HR, I,22; } \\
\text { IC de 95\%, } \\
\text { o,68-2,16). }\end{array}$ \\
\hline
\end{tabular}




\begin{tabular}{|c|c|c|c|c|}
\hline & $\begin{array}{l}\text { qualidade de } \\
\text { vida; } 5 \text { ) os } \\
\text { pacientes } \\
\text { relacionados } \\
\text { à } \\
\text { eletroconvuls } \\
\text { oterapia, } \\
\text { em geral, têm } \\
\text { uma } \\
\text { percepção } \\
\text { positiva do } \\
\text { tratamento. }\end{array}$ & & & \\
\hline Conclusão & $\begin{array}{l}\text { A ECT está } \\
\text { sendo um } \\
\text { tratamento } \\
\text { altamente } \\
\text { eficaz em } \\
\text { pacientes } \\
\text { com } \\
\text { depressão } \\
\text { resistente. } \\
\text { Ademais, } \\
\text { pode-se dizer } \\
\text { que tornou-se } \\
\text { um } \\
\text { procedimento } \\
\text { ainda } \\
\text { mais seguro e } \\
\text { útil tanto } \\
\text { para a fase } \\
\text { aguda, quanto } \\
\text { para a } \\
\text { prevenção de } \\
\text { novos } \\
\text { episódios } \\
\text { depressivos. }\end{array}$ & $\begin{array}{l}\text { Concluíram ser a } \\
\text { ECT uma opção } \\
\text { eficiente para } \\
\text { tratamento da } \\
\text { depressão, } \\
\text { porém, mais } \\
\text { estudos não } \\
\text { foram } \\
\text { descartados. }\end{array}$ & $\begin{array}{l}\text { Conclui-se que } \\
\text { a ECT deve ser } \\
\text { uma terapia de } \\
\text { primeira linha } \\
\text { para a } \\
\text { depressão } \\
\text { devido aos seus } \\
\text { profundos } \\
\text { efeitos no } \\
\text { alívio do } \\
\text { desespero em } \\
\text { certas } \\
\text { situações. }\end{array}$ & $\begin{array}{l}\text { Os resultados } \\
\text { deste estudo de } \\
\text { coorte apóiam o } \\
\text { uso contínuo de } \\
\text { ECT } \\
\text { para reduzir o } \\
\text { risco de suicídio } \\
\text { em pacientes } \\
\text { hospitalizados que } \\
\text { estão gravemente } \\
\text { deprimidos, } \\
\text { especialmente } \\
\text { aqueles que estão } \\
\text { com mais de } 45 \\
\text { anos e com } \\
\text { subtipo psicótico. }\end{array}$ \\
\hline
\end{tabular}

Tabela 3. Síntese dos estudos e seus principais resultados

\begin{tabular}{|c|c|c|}
\hline Autor/Ano. & $\begin{array}{l}\text { Cao et al., } \\
(2018)\end{array}$ & $\begin{array}{c}\text { Scherer e Scherer } \\
(\text { (201I) }\end{array}$ \\
\hline Objetivo & $\begin{array}{l}\text { Investigar as } \\
\text { alterações } \\
\text { volumétricas } \\
\text { dos } \\
\text { subcampos }\end{array}$ & $\begin{array}{c}\text { Descrever a } \\
\text { participação da } \\
\text { equipe no } \\
\text { procedimento de } \\
\text { eletroconvulsoter }\end{array}$ \\
\hline
\end{tabular}




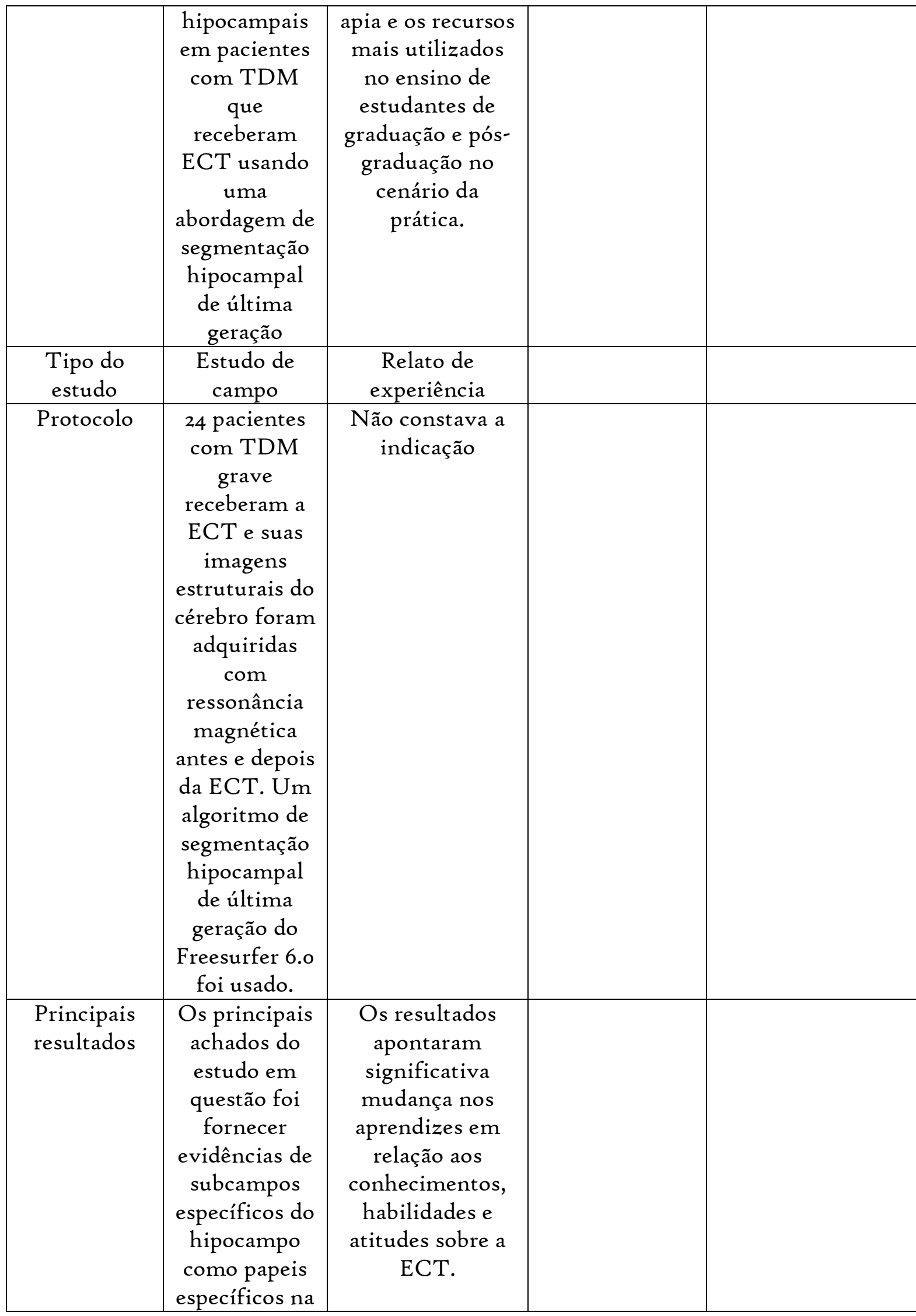




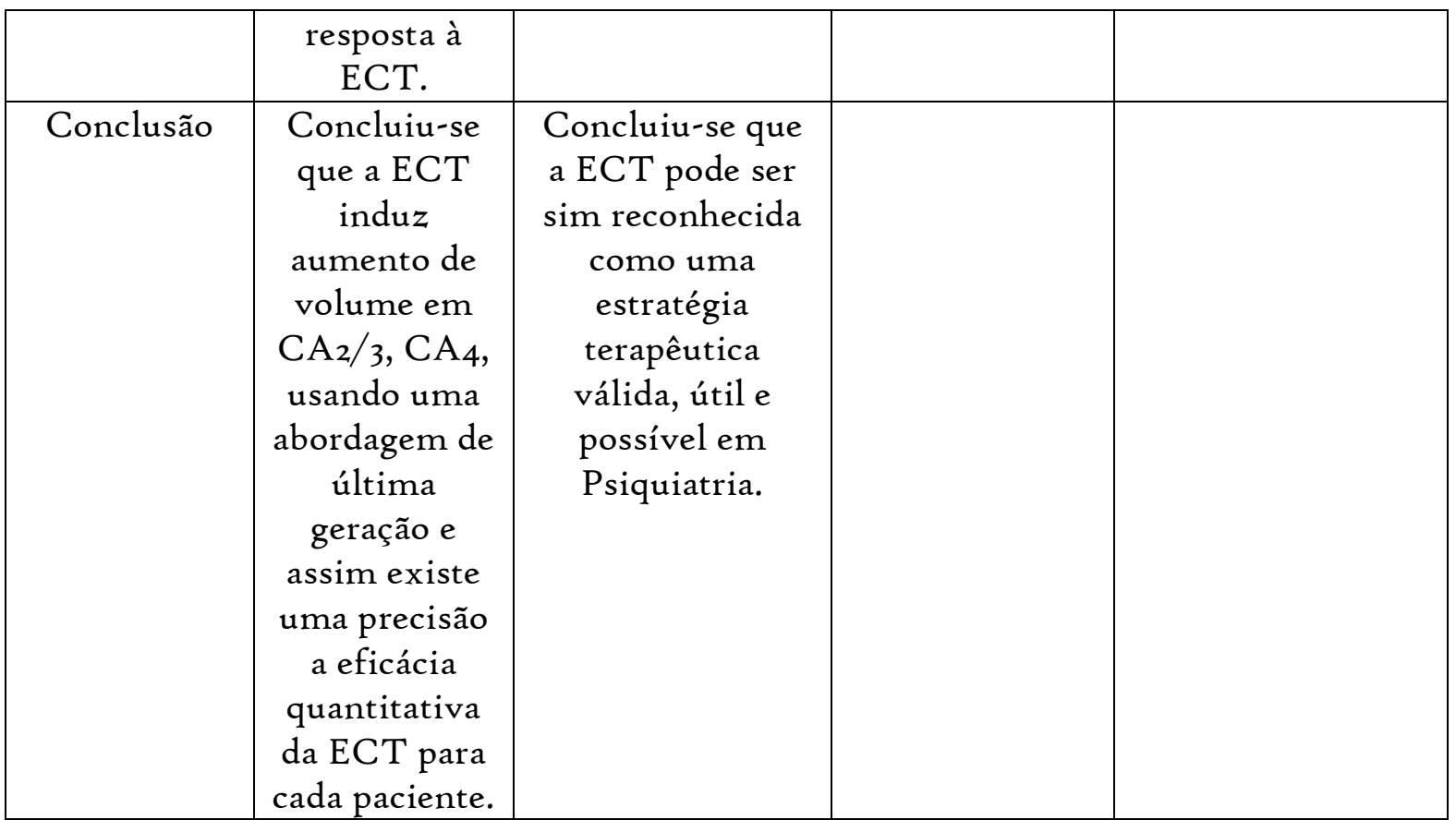

\section{DISCUSSÃO}

Com relação à problemática ora elencada, qual seja, importância da utilização da

ECT no tratamento da depressão, verificou-se, inicialmente, que apesar alvo de polêmicas e divergências em relação à sua eficácia tem mostrado o contrário de tal posicionamento. O primeiro estudo analisado, mostrou bem claramente a melhora significativa nos sintomas depressivos maiores a partir do tratamento com a ECT. Ademais, os resultados apontaram que ela pode ser administrada com segurança na melhora de tais episódios depressivos, inclusive quando há comorbidade, tal como era o relato de caso em questão que o paciente apresentava (RAPINESI, et al., 2013).

O segundo estudo analisado, apesar de se tratar de uma revisão bibliográfica, trouxe também contribuição tendo em vista ser proveniente de uma revisão integrativa e apresentar como conclusões que a partir de um modelo assistencial colaborativo, diferentes especialidades médicas devem trabalhar em conjunto para elaborar recomendações de prática abrangentes que visem modificar a técnica da ECT de acordo com cada população especial. Referido estudo ainda preconizou que apesar dos olhares estigmatizantes no decorrer dos anos, a ECT ela evoluiu no tratamento da depressão e tende a evoluir ainda mais. 
Ma et al., (2020) verificaram limitações no estudo quanto ao tamanho das amostras ( $\mathrm{n}$ pequeno) atrelado ainda que ao fator que todos os indivíduos eram pacientes internados, porém, ainda que com tais limitações, referido estudo de pacientes identificados em um hospital onde a ECT era muito mais comumente usada do que na maioria dos hospitais dos Estados Unidos sugere que a ECT pode ser eficaz em pacientes com sintomas graves, mas que não se qualificam formalmente como resistentes ao tratamento.

Ainda apresentou eficácia da ECT no tratamento dos sintomas da depressão o estudo realizado por Santos Júnior et al., (2013) apresentando a ECT foi positiva (boa / excelente) na maioria dos casos $(89,7 \%)$, reforçando a importância deste método terapêutico no tratamento de transtornos mentais graves. Este achado está em concordância com outros estudos recentes que mostram que a ECT, quando bem aplicado e indicado com precisão, é eficaz e seguro, e pode levar a melhorias na qualidade de vida através da rápida mitigação dos sintomas. Apesar de seu grande valor clínico, a ECT ainda é subestimada em Políticas públicas de saúde brasileiras, por preconceito e equívocos espalhados pela mídia e pela imprensa leiga.

A partir de metanálises desenvolvidas Antunes et al., (2019) verificaram certa superioridade da ECT em relação a outros tipos de tratamento, inclusive o medicamentoso. Porém. Não foi encontrada diferença significativa na realidade, nem na velocidade de ação da ECT quando comparadas às formas de onda sinusoidal (nove estudos) e pulso breve (Io estudos), havendo sobreposição dos intervalos de confiança. Esse estudo sugeriu que a ECT é superior na velocidade de ação no subgrupo de pacientes com depressão psicótica. Também apontaram que sua eficácia depende muito da técnica usada.

Antunes et al., (2019) verificaram que o tratamento com a ECT é descontinuado após alcançado êxito. Por tal motivo, os autores sugerem que a manutenção deve ser feita e também deve ser individualizada. De maneira geral, os estudos analisados pelos autores, verificaram ser de significativa importância a utilização da ECT na depressão, pois seus benefícios vão além da melhora sintomática. Há também melhora na $Q V$, funcionamento e bem-estar dos pacientes. Além disso, os pacientes que passam por essa experiência são, na maioria deles, favoráveis e apresentam atitudes positivas em relação à ECT. 
Inclusive foi oportunizada a análise do uso de ECT em adolescentes. A eletroconvulsoterapia (ECT) tem sido usada para o tratamento de doenças psiquiátricas em crianças e adolescentes há mais de 70 anos, porém, para a fim de que essa prática aconteça alguns critérios devem ser atendidos. A eletroconvulsoterapia foi relatada como altamente eficaz em $44 \%$ e moderadamente eficaz em $33 \%$ de todos os adolescentes pacientes; as taxas de resposta foram semelhantes às observadas em adultos (ZHAND et al., 2015)

A ECT, eventualmente, respondeu à farmacoterapia, porém, o resultado sugere que a falta de resposta à ECT não implica falta de resposta a outras opções de tratamento. $\mathrm{O}$ estudo ainda apontou alta taxa de efeitos adversos transitórios, incluindo dor de cabeça, dor muscular e náuseas/vômitos. As limitações deste estudo incluem número ínfimo da amostra e ausência de grupo controle (ZHAND et al., 2015).

Indo de encontro ao já analisado até então foi o e estudo realizado por Li et al., (2020) apontando que embora a ECT tenha mostrou ser inofensivo e eficaz, a taxa de aplicação ainda está baixo e é considerado o último recurso no tratamento depressão.

No mesmo sentido de uso benéfico, foi o achado de Rönnqvist et al., (2021) descobrindo a partir de suas investigações que a ECT para depressão estava associada com um risco reduzido de suicídio dentro de 3 e 12 meses de internação em comparação com não-ECT. A associação foi significativa em várias, mas não em todas as análises. Além disso, a ECT foi associada com reduziu a mortalidade por todas as causas dentro de 3 e 12 meses de internação em comparação com a não-ECT. Os principais achados do estudo em questão apresentaram evidências de subcampos específicos do hipocampo como papeis específicos na resposta à ECT (CAO et al. 2018).

A partir do trabalho com a equipe, foi possível uma orientação e esclarecimentos afincados sobre a ECT e em seguida participaram juntamente com a equipe de assistência. Observaram e acompanharam a equipe nos cuidados pós-procedimento. Os alunos manifestaram surpresa ao virem respostas exitosas após tratamento com a ECT (SCHERER; SCHERER, 20II). 


\section{CONCLUSÃO}

Concluiu-se que a depressão é problema de saúde pública e acomete, inclusive, o transtorno depressivo maior (TDM) já é considerado uma das principais doenças incapacitantes do mundo, afetando aproximadamente 350 milhões de pessoa. É importante considerar o contexto de vida sob a perspectiva histórica do paciente, pois as reações emocionais atuais podem estar diretamente relacionadas às vivências acumuladas no decorrer de toda a sua existência.

A partir dos resultados apresentados e discutidos verificou-se que a ECT quando usada com cautela e realizada por profissional competente, a ECT é uma opção de tratamento muito eficaz e deve ser disponível para pacientes selecionados. $O$ único desafio apresentado a partir da análise feita é lidar com o estigma, preconceito e resistência ao uso desse tratamento tão eficaz.

A limitação do presente estudo foi com relação ao número ínfimo de estudo na língua corrente a fim de uma análise mais afincada sobre a sua eficácia.

\section{REFERÊNCIAS}

ANTUNES, P.B. et al. Eletroconvulsoterapia na depressão maior: aspectos atuais. Rev Bras Psiquiatr., v.3I, Supl I):S26-33, 2019. Disponível em: https://www.scielo.br/j/rbp/a/5S $75 \mathrm{ctx} \mathrm{G}_{4} \mathrm{FFFVmdFCLYTkBz} /$ ?format=pdf\&lang=pt Acesso em: Io ago. 2021.

ARAUJO, F.A.F.M.de; NAKANO, T. de C.; GOUVEIA, M.L. de A. Prevalência de depressão e ansiedade em detentos. Aval. psicol., Porto Alegre,v.8,n. 3, p. 38I390, dez. 2009. Disponível

em $<$ http://pepsic.bvsalud.org/scielo.php?script=sci_arttext\&pid=Si67704712009000300oIo\&lng=pt\&nrm=iso $>$. Acesso em: 21 ago.

ASSIS, T.S.M. de. Eletroconvulsoterapia para o tratamento da depressão refratária à medicação: uma revisão sistemática. Revista Brasileira de Neurologia e Psiquiatria, v.24, n.I, p.4-I3, 2020. Disponível em: https://www.revneuropsiq.com.br/rbnp/article/view/497/202 Acesso em: 20 ago. 202I. 
BAUER et al. Diretrizes da World Federation of Societies of Biological Psychiatry (WFSBP) para tratamento biológico de transtornos depressivos unipolares, 2 parte: tratamento de manutenção do transtorno depressivo maior e tratamento dos transtornos depressivos crônicos e das depressões subliminares. Archives of Clinical Psychiatry (São Paulo), 36 (Suppl. 2), 58-76.

$\mathrm{CAO}, \mathrm{B}$. et al. Predicting individual responses to the electroconvulsive therapy with hippocampal subfield volumes in major depression disorder. Scientifics Reports, v.8, n. $5434,2018$.

HERMIDA, A. P. et al. Electroconvulsive Therapy in Depression. Psychiatr Clin N Am, v.4I, n.3, p.34I-353, 2018.

LI, M. et al. Effects of Electroconvulsive Therapy on Depression and Its Potential Mechanism. Frontiers in Psychology, v.II, n.8o, 2020.

MACHADO, F.B. et al. Eletroconvulsoterapia: implicações éticas e legais. Rev. Cient. Sena Aires. v.7, n.3, p.235-247, 2018. Disponível em: http://revistafacesa.senaaires.com.br/index.php/revisa/article/view/326/237 Acesso em: IO ago. 202I.

MATOS, A.C.S.; OLIVEIRA, I.R. de. Terapia cognitivo-comportamental da depressão: relato de caso. Rev. Ciênc. Méd. Biol, Salvador, v.6, n.12, 512-519. 2013. Disponível em: https://periodicos.ufba.br/index.php/cmbio/article/view/9203 Acesso em: 20 ago. 2021.

MUNIZ, K.R. et al. Eletroconvulsoterapia no tratamento da depressão refratária. In: SILVA NETO, B.R. et al., (org). Comunicação científica e técnica em medicina. Ponta Grossa/PR: Atena, 2020, p.8o-86.

OLIVEIRA, W.F. de. Eletroconvulsoterapia (ECT)/Eletrochoque: a produção de evidências sobre seu uso, eficácia e eficiência. Cadernos Brasileiros de Saúde Mental, v.II, n.28, p.46-68, 2019. Disponível em: https://periodicos.ufsc.br/index.php/cbsm/article/view/69772 Acesso em: 20 ago. 202I.

PALOSKI, L.H.; CHRIST, H.D. Terapia cognitivo-comportamental para depressão com sintomas psicóticos: uma revisão teórica. Contextos Clínic, São Leopoldo, v. 7, n. 2, p. 220228, dez. 2014. Disponível em <http://pepsic.bvsalud.org/scielo.php?script=sci_arttext\&pid=Si983$34822014000200010 \& \operatorname{lng}=\mathrm{pt} \& \mathrm{nrm}=\mathrm{iso}>$. Acesso em: 20 ago. 2021. 
RAPINESI, C. et al. Effectiveness of Electroconvulsive Therapy in a Patient With a Treatment-Resistant Major Depressive Episode and Comorbid Body Dysmorphic Disorder. Journal of ECT, v.29, n.2, 2013.

ROCHA, Glaucia Mitsuko Ataka da et al. Psicoterapia breve psicodinâmica de caso grave de depressão em serviço-escola: limites e alcance. Contextos Clínic, São Leopoldo, v.9,n.I,p. 86-97,jun.2016. Disponível em $<$ http://pepsic.bvsalud.org/scielo.php?script =sci_arttext\&pid=Si983348220160ooroooo8\&lng=pt\&nrm=iso $>$. Acesso em: 20 ago. 2021.

RÖNNQVIST, I. et al. Electroconvulsive Therapy and the Risk of Suicide in Hospitalized Patients With Major Depressive Disorder. JAMA Network Open. v.4, n.7, 2021;4(7):e2116589.

SCHERER, A.P.; SCHERER, E.A. Eletroconvulsoterapia em idosos deprimidos: relato de experiência. Cogitare Enfermagem, v.16, n.2, p.365-368, 20II. Disponível em: https://revistas.ufpr.br/cogitare/article/view/21819/14229 Acesso em: Acesso em: 20 ago. 202I.

SOUZA, I.C.W.de; CANDIDO, C.F.G. Diagnóstico psicológico e terapia cognitiva: considerações atuais. Rev. bras.ter. cogn., Rio de Janeiro, v.5, n.2, p.82-93, nov.2009. Disponível em 〈http://pepsic.bvsalud.org/scielo.php?script=sci_arttext\&pid=Sı8o8$56872009000200009 \& \operatorname{lng}=$ pt\&nrm=iso $>$. Acesso em: 20 ago. 2021.

ZHAND, N. et al. Use of Electroconvulsive Therapy in Adolescents With Treatment-Resistant Depressive Disorders: A Case Series. Journal of ECT, v.31, n.4, 2015. 